\title{
Oxidative Stress Index Predicts the Severity of COVID-19
}

\author{
Harold I. Zeliger and Harvey Kahaner
}

\begin{abstract}
We have hypothesized that the Oxidative Stress Index (OSI) can be used to predict the severity of COVID-19. The recently published Clinical Risk Score Calculation (CRSC) based upon clinical data ascertained at the time of hospital admission for patients in $\mathbf{5 7 5}$ hospitals in China following the COVID-19 outbreak confirms and validates our hypothesis.
\end{abstract}

Index Terms - COVID-19, Oxidative Stress Index, COVID19 severity.

\section{INTRODUCTION}

We have hypothesized that the Oxidative Stress Index (OSI) can be used to predict the severity of COVID-19 [1]. A recently published clinical study describing a numerical clinical risk score to predict COVID-19 severity prediction Liang, et al. [2] provides confirmation and validation of our hypothesis. Though Liang, et al. do not specifically address oxidative stress (OS) or OSI, all the parameters shown by them to increase COVID-19 severity are known to elevate OS and raise the OSI. Here, we demonstrate how the clinically supported results reported in the CRSC paper precisely dovetail with our hypothesis and lend credence to the use of the OSI to predict COVID-19 severity.

\section{METHOD}

The method used is a comparison of each of the parameters used in our hypothesis with those used in the CRSC to demonstrate the application of a non-invasive, mathematical way to predict of COVID-19 severity and the validity by the OSI.

\section{RESULTS}

Our hypothesis describes how OS can be used to predict the severity of COVID-19 and how the eight parameters making up the OSI can be used to mathematically predict disease severity. As discussed here, the parameters used in the CRSC, which mathematically predict COVID-19 severity, are all incorporated into the eight OSI items.

\section{A. Oxidative Stress and Disease}

Elevated oxidative stress has been shown to be directly related to the likelihood of falling ill with non-

Published on July 13 30, 2020.

Harold I. Zeliger,Ph. D., Zeliger Research, LLC, USA

(corresponding e-mail: hiz ${ }^{\circledR}$ zeliger.com).

Harvey Kahaner, M. D., Zeliger Research, LLC, USA. communicable disease, as well as with the onset, frequency and severity of infectious viral, bacterial, fungal and parasite-carried diseases. These enhanced effects have been shown to prevail in an OS induced dose response relationship no matter what the cause(s) of OS elevation. OS-raising causes can include age, gender, environmental exposures toxic chemicals and radiation, diet, smoking, alcohol consumption and other lifestyle choices, pre-existing disease, disease symptoms, medications regularly taken and chronic psychological stress as well as combinations of these [3].

\section{B. Oxidative Stress Index}

The Oxidative Stress Index, based on a questionnaire that takes all OS elevated factors into account, has been shown to be indicative of OS level and a predictor of the likelihood of disease onset, as well as of disease severity [4]. Though originally proposed as a $400+$ item questionnaire, the OSI has been modified to an eight-item condensed format [5] and subsequently proposed as a predictor of severity of COVID-19 [1].

\section{Clinical Risk Score Calculation (CRSC)}

Recently, a clinical risk score calculation has been developed. The CRSC shows that one's pre-existing conditions at the time of presentation with COVID-19 are indicative of ultimate disease severity in a mathematically related matter. A calculation tool for this prediction as well as supporting validation data are presented [2]. The CRSC is based upon age, lifestyle, pre-existing conditions and clinical data ascertained at the time of hospital admission for patients in 575 hospitals in China following the COVID-19 outbreak. This study shows that such data can be used to predict the severity of COVID-19.

\section{Comparison of the OSI and the CRSC}

Both the OSI and CRSC demonstrate that a patient's health and lifestyle data can be used to predict COVID-19 severity and that predictions regarding severity can be mathematically obtained. Though the CRSC does not relate relevant disease severity factors to oxidative stress, all the factors that enter into the CRSC calculation (age, detrimental lifestyle choices pre-existing diseases and the symptoms associated with these) have elevated oxidative stress associated with them. The CRSC demonstrates that higher numerical scores are mathematically related to disease severity. Generating OSI scores from the results obtained in the CRSC shows that higher scores of OSI directly correspond to increases in the CRSC.

In lieu of clinical evaluations, the OSI uses eight parameters, all of which elevate OS and have been shown to incorporate the clinical parameters relied upon by the CRSC 
[4]. In addition, the OSI includes environmental and lifestyle parameters not included in the CRSC.

The OSI parameters include:

- Age;

- Gender, height and weight;

- Pre-existing disease(s);

- Medications regularly taken;

- Genetics;

- Socioeconomic status as indicated by education level;

- Residence as an indicator of pollution exposure;

- Chronic psychological stress.

By incorporating all the above, the OSI not only predicts the probability of COVID-19 severity in coronavirusexposed individuals, but also illustrates why some young people who are exposed to the virus develop more severe symptoms than their peers.

\section{DISCUSSION}

The CRSC addresses clinically measured parameters for those already afflicted with COVID-19 and generates a numerical data point which can be used to predict disease severity. The OSI can be used in this manner as well. It not only also provides a basis for predicting an ill individual's propensity for disease severity but can also be used to predict the propensity for COVID-19 onset prior to the manifestation of disease symptoms. The OSI can thus be used to identify vulnerable individuals of all ages and backgrounds and can also be indicative of disease prevention steps to be taken. The CRSC is a valuable clinical tool that has great applicability in preparing clinicians to treat those presenting with the disease. The OSI can be applied to seemingly healthy people as well as those who are ill with COVID-19. As has been previously shown, the OSI also lends itself to the likelihood of the onset of other diseases as well and can be used as a health screening tool in areas with limited medical facilities and personnel [4].

\section{CONCLUSION}

The OSI has previously been hypothesized to predict the severity of COVID-19 [1]. The recently published CRSC provides data that confirm and validate the OSI mathematical approach to predicting COVID-19 severity.

\section{REFERENCES}

[1] H. I. Zeliger, H. Kahaner, "Can the Oxidative Stress Index predict the severity of COVID-9?", Eur J Med Health Sci, vol. 2, issue 2, 2020, DOI: http://dx.doi.org//10.24018/ejmed.2020.2.2.233.

[2] W. Liang, H. Liang, L. Ou, B. Chen, A. Chen, L. Caichen, et al., "Development and validation of a clinical risk score to predict the occurrence of critical illness in hospitalized patients with COVID19”, JAMA Intern Med., May 12, 2020 Doi:10.1001/jamainternmed.2020.2033.

[3] H. I. Zeliger, "Predicting disease onset in clinically health people", Intediscip Toxicol, vol. 9(2), pp. 39-54, 2016.

[4] H. I. Zeliger, "Oxidative stress index predicts disease onset", J Med Res Pract, vol. 6(3), pp. 86-92, 2017.

[5] H. I. Zeliger, "Oxidative stress index (OSI) condensed questionnaire", Eur J Med Health Sci, vol. 2, issue 1, 2020, DOI http://dx.doi.org//10.24018/ejmed.2020.2.1.163. 